\title{
BOVINE ENDOTOXICOSIS - ASPECTS OF RELEVANCE TO PRODUCTION DISEASES. A REVIEW
}

\author{
Pia Haubro Andersen \\ Dept. Clinical Studies, Section of Surgery. Royal Veterinary and Agricultural University \\ DK-1870 Frederiksberg Denmark
}

Endotoxins are structural parts of the Gram negative bacteria membrane. Endotoxins are extremely toxic to cattle in parenteral doses in the range $100 \mathrm{ng}$ per $\mathrm{kg}$ bw. The development of endotoxicosis is believed to play a significant role in the pathogenesis of various clinical conditions associated with production diseases, from ruminal acidosis and "sudden death syndrome" in feed-lot cattle, to laminitis in dairy cattle, Gram negative infections, shock and death. Decisive evidence for the presence of endotoxemia in any of these conditions is relatively scarce, but circumstantial evidence is more common, because in many respects the clinical and biochemical symptoms of bovine endotoxicosis - gastrointestinal stasis, diarrhoea, hypocalemia, hypozinemia and leukopenia - are similar to those observed in various of the production diseases mentioned. This review will attempt to discuss the possible sources of endotoxin in the bovine clinic, the exposure routes and the relevance of endotoxin for the development of feeding related production diseases. Some aspects regarding individual susceptibility will be discussed as well.

The source of endotoxin is mainly Gram negative infection and or gastrointestinal contents. The exposure routes are infected organs (udder, uterus) but exposure may also take place via translocation through a leaky gastrointestinal barrier. Large quantities of endotoxin are present in the rumen of cattle, and the concentration increases when concentrate is added to a hay diet. When ruminitis is present, the ruminal epithelium may become leaky. In experimentally induced ruminal acidosis endotoxin can be detected in the pre-hepatic and sometimes in the systemic circulation. The endotoxin concentrations of the systemic blood were lower than the pre-hepatic concentrations, demonstrating the clearance effect of the liver. Another study has shown that cows with hepatic lipidosis are relatively incapable of clearing injected endotoxin, when compared to cows with normal livers. Such studies underline the significance of the bovine fatty liver syndrome in the production disease complex.

Large variations in susceptibility of cattle to endotoxin are commonly reported and the causative factors are extremely interesting. Failure to detect endotoxins in the blood of clearly sick cows has frustrated many researchers and it has been questioned whether the presence of endotoxemia is necessary for the development of endotoxicosis. With the increased knowledge of the links between immunology, stress, parturition and ocurrence of production diseases, it becomes increasingly important to appreciate that the toxicity of endotoxin is caused by the response of the host organism, rather than by the properties of endotoxin per se. Differences in the bovine response to endotoxin may therefore equally well be ascribed to differences in the immunological line of defense as to factors related to the endotoxin of an invading Gram negative microorganism.

Investigation of these interrelationships may provide a basis for the identification of indicators of health which can be used in future screenings both for herd-health and genetically determined conditions. 